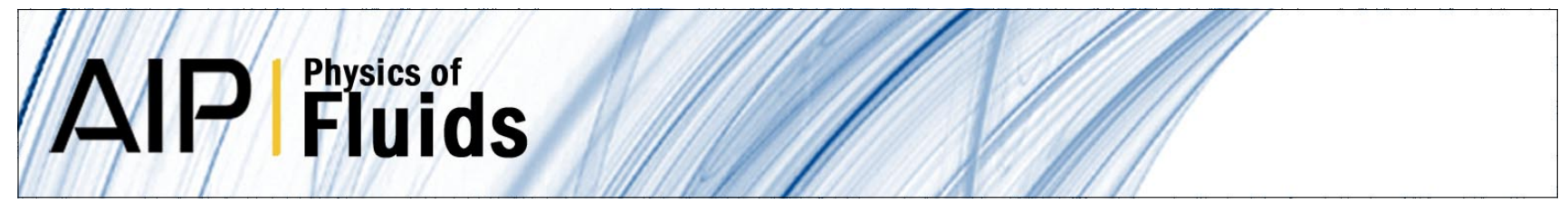

\title{
A streamwise-constant model of turbulent pipe flow
}

Jean-Loup Bourguignon and Beverley J. McKeon

Citation: Phys. Fluids 23, 095111 (2011); doi: 10.1063/1.3640081

View online: http://dx.doi.org/10.1063/1.3640081

View Table of Contents: http://pof.aip.org/resource/1/PHFLE6/v23/i9

Published by the American Institute of Physics.

\section{Related Articles}

On the spectra of nozzle-exit velocity disturbances in initially nominally turbulent, transitional jets Phys. Fluids 23, 091702 (2011)

Heat transfer and friction factor inside elliptic tubes fitted with helical screw-tape inserts J. Renewable Sustainable Energy 3, 023110 (2011)

Visualizing the very-large-scale motions in turbulent pipe flow

Phys. Fluids 23, 011703 (2011)

Lorentz force velocimetry based on time-of-flight measurements

Phys. Fluids 22, 125101 (2010)

Shear-flow excitation mechanisms of recessed localized arc-filament plasma actuators

Phys. Fluids 22, 116103 (2010)

\section{Additional information on Phys. Fluids}

Journal Homepage: http://pof.aip.org/

Journal Information: http://pof.aip.org/about/about_the_journal

Top downloads: http://pof.aip.org/features/most_downloaded

Information for Authors: http://pof.aip.org/authors

\section{ADVERTISEMENT}

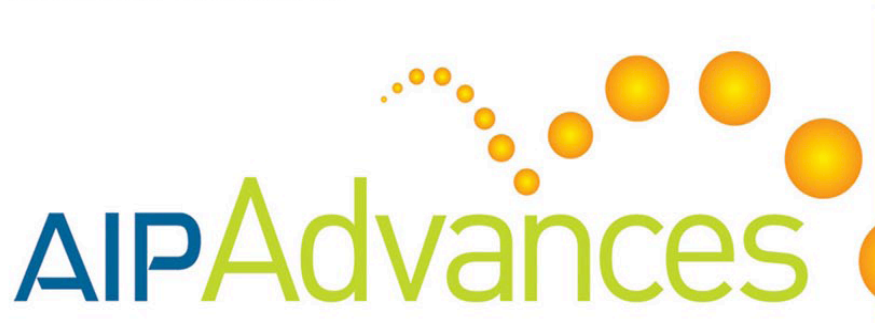

Submit Now

Explore AIP's new

open-access journal

Article-level metrics now available

Join the conversation!

Rate \& comment on articles 


\title{
A streamwise-constant model of turbulent pipe flow
}

\author{
Jean-Loup Bourguignon and Beverley J. McKeon \\ Graduate Aerospace Laboratories, California Institute of Technology, Pasadena, California 91125, USA
}

(Received 4 June 2011; accepted 16 August 2011; published online 29 September 2011)

\begin{abstract}
A streamwise-constant model is presented to investigate the basic mechanisms responsible for the change in mean flow occuring during pipe flow transition. The model is subject to two different types of forcing: a simple forcing of the axial momentum equation via a deterministic form for the streamfunction and a stochastic forcing of the streamfunction equation. Using a single forced momentum balance equation, we show that the shape of the velocity profile is robust to changes in the forcing profile and that both linear non-normal and nonlinear effects are required to capture the change in mean flow associated with transition to turbulence. The particularly simple form of the model allows for the study of the momentum transfer directly by inspection of the equations. The distribution of the high- and low-speed streaks over the cross-section of the pipe produced by our model is remarkably similar to one observed in the velocity field near the trailing edge of the puff structures present in pipe flow transition. Under stochastic forcing, the model exhibits a quasiperiodic self-sustaining cycle characterized by the creation and subsequent decay of "streamwiseconstant puffs," so-called due to the good agreement between the temporal evolution of their velocity field and the projection of the velocity field associated with three-dimensional puffs in a frame of reference moving at the bulk velocity. We establish that the flow dynamics are relatively insensitive to the regeneration mechanisms invoked to produce near-wall streamwise vortices, such that using small, unstructured background disturbances to regenerate the streamwise vortices in place of the natural feedback from the flow is sufficient to capture the formation of the high- and lowspeed streaks and their segregation leading to the blunting of the velocity profile characteristic of turbulent pipe flow. We propose a "quasi self-sustaining process" to describe these mechanisms. (C) 2011 American Institute of Physics. [doi:10.1063/1.3640081]
\end{abstract}

\section{INTRODUCTION}

Linear models of pipe flow, e.g., Schmid and Henningson $(1991),{ }^{1}$ have been found to capture general characteristics of the coherent structures present in turbulent flow but are unable to reproduce the change in mean flow associated with transition to turbulence. Linear studies have shown that linear non-normality in subcritical shear flows is required to sustain turbulence (Henningson and Reddy, 1994) ${ }^{2}$ and so are the terms linear in turbulence fluctuations (Kim and Lim, 1993). ${ }^{3}$ The non-normality of the linear Navier-Stokes (LNS) operator results in large amplification of disturbances and transient growth of initial perturbations. However, the growth of the most amplified structures modify the mean flow in a way that reduces the amplification potential, i.e., the non-normality. ${ }^{4}$ Hence, realistic models for pipe flow transition must include mechanisms leading to a change in mean flow as the perturbations develop.

Since the blunting of the velocity profile is accurately captured by the full Navier-Stokes equations (NSE), a convenient approach is to go back to the NSE and invoke simplifying assumptions in order to reduce the complexity of the model instead of starting from the LNS and adding some sort of nonlinearity, e.g., Bagget et al. (1995). ${ }^{5}$ In this work, we focus on the simplification associated with considering a projection of the NSE onto the streamwise direction or streamwise-constant models which retain a nonlinear term.
Streamwise-constant models describe the evolution of the three components of velocity in a plane perpendicular to the mean flow and are equivalently referred to as $2 \mathrm{D} / 3 \mathrm{C}$. A streamwise-constant model for fully developed (pipe) flow was derived by Joseph and Tao $(1963)^{6}$ and shown to be globally stable for all Reynolds numbers. ${ }^{7}$ Thus, the 2D/3C model has the useful property of having a unique fixed point corresponding to the laminar flow. A stochastically forced $2 \mathrm{D} / 3 \mathrm{C}$ model formulated in terms of a cross-stream streamfunction and the deviation of the streamwise velocity from the (linear) laminar profile was used by Gayme et al. $(2010)^{8}$ to study Couette flow and successfully captured both the blunting of the velocity profile and structures similar to the streamwise-elongated vortices and streaks observed in experiments. In general terms, the stochastically forced 2D/3C model exploits the large amplification of background disturbances due to the non-normality of the linearized operator described by Farrell and Ioannou $(2000)^{9}$ which has been shown to reach a maximum for streamwise-constant disturbances (Bamieh and Dahleh, 2001). ${ }^{10}$ The latter authors showed that streamwise-constant disturbances are amplified proportionally to $R e^{3}$ vs. $R e^{3 / 2}$ for streamwise-variant disturbances. In addition, Jovanovic and Bamieh ${ }^{11}$ demonstrated that the largest amplification is only obtained by forcing in the plane perpendicular to the mean flow and is observed in the streamwise velocity component. 
Pipe flow is well suited for an assumption of streamwise invariance since streamwise-elongated coherent structures have been shown to play an important role during transition, e.g., Ref. 12, as well as in fully developed turbulence, e.g., Refs. 13-17. The streamwise-elongated coherent structures in pipe flow, both in the near-wall region and further from the wall, take a form dominated by quasi-streamwise vortices and streaks of streamwise velocity. A body of recent work in the literature suggests a connection between these features and studies of the LNS. For example, the most (temporally) amplified mode of the LNS, based on an energy norm, is streamwise-constant with an azimuthal wavenumber $n=1$ and features a pair of counter-rotating vortices which create streaks by convecting streamwise momentum. ${ }^{1}$ Reshotko and Tumin $(2001)^{18}$ studied the spatial evolution of optimal disturbances in pipe flow in contrast to previous studies focusing on the temporal evolution, arguing that a spatial study is better suited for comparison with experiments in which the disturbances are growing as they convect downstream. They concluded that the most amplified disturbances are stationary and have an azimuthal wavenumber $n=1$.

Additionally, important support for the streamwiseconstant model comes from the nonlinear study of turbulent Couette flow by Reddy and Ioannou (2000), ${ }^{19}$ which emphasizes the dominant role played by the streamwise-constant modes in the flow dynamics. Based on an energy transfer analysis, the latter authors showed that the streamwiseconstant modes with azimuthal wavenumber $\pm n$, where $n$ is an integer, dominate energy extraction from the laminar base flow using linear non-normal mechanisms, and maintain the mean turbulent flow via their nonlinear interaction. Note that the mean turbulent mode does not extract energy directly from the laminar base flow.

The laminar base flow in a pipe which is linearly stable for all Reynolds numbers ${ }^{20,21}$ becomes unstable when streamwise-constant vortices and velocity streaks are superposed due to the creation of inflection points ${ }^{22}$ which sustain the growth of infinitesimal $3 D$ disturbances until the streaks decay. ${ }^{23}$ Waleffe $(1997)^{4}$ argued that the $3 D$ disturbances can regenerate the vortices, or "rolls," by nonlinear interaction which consequently create the streaks by convecting streamwise momentum, leading to a self-sustaining process (SSP) which occurs across a range of shear flows. The regeneration mechanisms invoked by Waleffe were later revisited by Schoppa and Hussain (2002), ${ }^{24}$ who attributed the regeneration of the rolls to a mechanism involving transient growth of the streaks. In this model, the $3 D$ infinitesimal perturbations exhibit transient growth and evolve into sheets of streamwise vorticity which are then stretched by the mean shear and collapse, resulting in the formation of streamwise rolls. The SSP was shown to dominate the near-wall cycle in fully developed turbulence and features of the SSP are also observed in turbulent puffs occurring during pipe flow transition (van Doorne and Westerweel, 2009 25 ) and in the edge state analysis of Schneider et al. (2007), ${ }^{26}$ an alternate view of the approach to turbulence associated with the treatment of the turbulent state as a chaotic saddle in state space.

Traditionally, the later stages of transition to turbulence in pipe flow have been characterized by the creation of puffs and slugs (Wygnanski and Champagne, $1973^{27}$ ). Puffs have been identified as the flow response to large amplitude disturbances at low Reynolds number, e.g., $R e \approx 2000$, and are characterized by a sharp trailing edge and a smooth leading edge whereas slugs are created by low amplitude disturbances at larger Reynolds number, $R e>3000$ and have sharp leading and trailing edges. ${ }^{27}$ The puffs are sustained via a SSP taking place near the trailing edge, characterized by the creation of low-speed streaks inside the puff which convect slower than the puff and create a shear layer at the boundary with the laminar flow at the back of the puff (Shimizu and Kida $\left.(2009)^{28}\right)$. The shear layer is subject to KelvinHelmholtz instability resulting in the creation of streamwise vortices by roll-up of vortex sheets. The streamwise vortices propagate faster than the puff and maintain the turbulence inside the puff as they re-enter it. The quasi-periodic generation of streamwise vortices near the trailing edge of the puffs, where the transition from laminar to turbulence takes place, was also reported by van Doorne and Westerweel (2009). ${ }^{25}$ Hof et al. (2010) ${ }^{29}$ suggested a new driving mechanism for puffs based on the formation of inflection points in the velocity profile near the trailing edge of the puff whose instability sustains turbulence inside the puff.

The clear distinction between puffs and slugs made by Wygnanski and Champagne ${ }^{27}$ was later questioned by Darbyshire and Mullin (1995) ${ }^{30}$ who observed mixed occurences of puffs and slugs. More recently, Duguet et al. (2010) $)^{31}$ argued that slugs are out-of-equilibrium puffs and, therefore, cannot exist together with stable equilibrium puffs, which are observed at $R e \approx 2200$ and convect slightly slower than the mean flow. Equilibrium puffs keep a constant length as they travel downstream and are separated by regions of laminar flow which are necessary to sustain them (as noticed by Lindgren, $1957,{ }^{32}$ see also Hof et al., 2010 ${ }^{29}$ ). In general terms, equilibrium puffs represent a minimal flow unit able to sustain turbulence. The particle-image-velocimetry (PIV) measurements of Hof et al. (2004) $)^{33}$ confirmed that the dominant flow structures inside a puff are quasi-streamwise vortices and streaks which are independent of the method used to generate the puff, ${ }^{27}$ and also highlighted the similarity between the traveling wave solutions of the NSE and the velocity field near the trailing edge of a puff. At larger Reynolds number, the puffs expand as they convect downstream and tend to merge together, becoming unstable via a KelvinHelmholtz instability of the wall-attached shear layers (Duguet et al., 2010 ${ }^{31}$ ) and resulting in the formation of slugs which keep expanding until the whole flow domain is dominated by turbulent motion.

In this paper, we present a streamwise-constant model for turbulent pipe flow along with an exploration of the simplest forcing models which allow us to isolate the basic mechanisms governing the dynamics that result in the blunting of the velocity profile. The model is described in Sec. II, together with the numerical methods employed to simulate the flow. In Sec. III, we use a simple, steady, deterministic forcing to isolate the effects of the linear and nonlinear terms, showing that the linear coupling between the in-plane and axial velocities leads to the formation of high- and lowspeed streaks (defined with respect to the laminar base flow) 
and that the nonlinear coupling convects the low-speed streaks towards the center of the pipe and the high-speed streaks towards to wall, resulting in the blunting of the velocity profile. In Sec. IV, we describe the response of our model to stochastic forcing in the cross-stream plane, demonstrating the generation of streamwise-constant versions of a puff. We conclude this study by summarizing the main achievements obtained with the 2D/3C model for pipe flow.

\section{DESCRIPTION OF THE MODEL AND NUMERICAL METHODS}

The streamwise-constant model of turbulent pipe flow is derived from the NSE written in cylindrical coordinates under the assumption of streamwise invariance, i.e., it constitutes a projection of the NSE onto the streamwise direction. We employ a nondimensionalization based on the pipe radius $R$ and the bulk velocity $\bar{U}$, i.e., $\eta=\frac{r}{R}, \tau=\frac{\bar{U} t}{R}$, and $R e=\frac{2 R \bar{U}}{\nu}$, where $\nu$ is the kinematic viscosity, throughout this paper. Continuity is enforced via the introduction of a dimensionless streamfunction $\Psi$ whose evolution equation is obtained by taking the curl of the NSE projected in the axial direction. The model consists of a forced evolution equation for the streamfunction, from which the radial and azimuthal velocities can be derived, and an evolution equation for the axial velocity (in terms of the deviation from the laminar profile) corresponding to the axial momentum balance, subject to boundary conditions (BCs) of no-slip and no-penetration on the wall of the pipe. The deviation of the local axial velocity from laminar illustrates how the flow evolves away from the laminar state and is defined as

$$
u_{x}(\eta)=\tilde{u}_{x}(\eta)-U(\eta)
$$

where $\tilde{u}_{x}$ is the instantaneous axial velocity and $U(\eta)=1-\eta^{2}$ is the laminar base flow.

The 2D/3C model was first derived by Joseph and Tao $(1963)^{6}$ and is written as follows for the cylindrical coordinate system shown in Figure 1:

$$
\left\{\begin{array}{l}
\frac{\partial \Delta \Psi}{\partial \tau}=\frac{2}{R e} \Delta^{2} \Psi+\mathcal{N}_{\psi} \\
\frac{\partial u_{x}}{\partial \tau}=C-\frac{1}{\eta} \frac{\partial \Psi}{\partial \phi} \frac{\partial U}{\partial \eta}-\frac{1}{\eta} \frac{\partial \Psi}{\partial \phi} \frac{\partial u_{x}}{\partial \eta}+\frac{1}{\eta} \frac{\partial \Psi}{\partial \eta} \frac{\partial u_{x}}{\partial \phi}+\frac{2}{R e} \Delta u_{x} \\
\left.\Psi\right|_{\eta=1}=\left.\frac{\partial \Psi}{\partial \eta}\right|_{\eta=1}=0
\end{array}\right.
$$

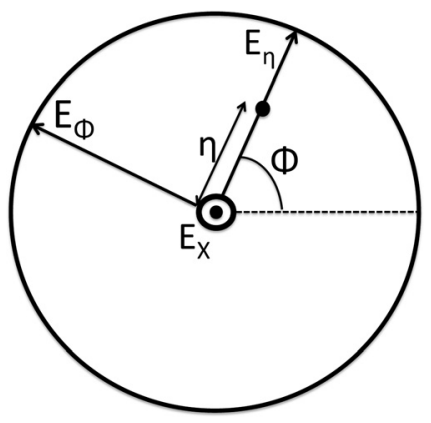

FIG. 1. The coordinate system used to project the Navier-Stokes equations. where $\Delta=\frac{1}{\eta}\left(\frac{\partial}{\partial \eta}\left(\eta \frac{\partial}{\partial \eta}\right)+\frac{1}{\eta} \frac{\partial^{2}}{\partial \phi^{2}}\right)$ is the $2 \mathrm{D}$ Laplacian. The radial and azimuthal velocities are defined by $u_{r}=\frac{1}{\eta} \frac{\partial \Psi}{\partial \phi}$ and $u_{\phi}=-\frac{\partial \Psi}{\partial \eta}$. Only the streamfunction equation is forced, based on the results of the study by Jovanovic and Bamieh $(2005)^{11}$ which showed that maximum amplification is obtained by forcing in the cross-sectional plane in the linearized NSE. Thus, $\mathcal{N}_{\psi}$ represents a forcing term that is required to maintain the perturbation energy in an otherwise stable system and can be considered to represent "noise" that is always present in experiments, e.g., wall roughness, vibrations, non-alignment of the different sections of the pipe, thermal effects, as well as taking into account the effects not modeled by the streamwise invariance approximation. In the subsequent sections, we consider two of the simplest possible forms for $\mathcal{N}_{\psi}$ in order to investigate the origin of the blunting of the mean velocity profile. The nonlinear terms in the governing equation for the streamfunction are neglected in order to obtain the simplest model able to capture the blunting of the velocity profile and also because their effects can be incorporated into the unstructured forcing term $\mathcal{N}_{\psi}$. Moreover, the study of Gayme et al. $(2011)^{8}$ showed that there are no significant differences in the Couette flow statistics obtained from the model based on a linearized streamfunction equation compared to the fully nonlinear $2 \mathrm{D} / 3 \mathrm{C}$ model. The bulk velocity is maintained constant by adjusting the pressure gradient $C$, i.e., the Reynolds number is held constant for each study. The streamwise velocity behaves as a passive scalar convected by the in-plane velocities.

The 2D/3C model with stochastic forcing is discretized using a spectral-collocation method based on Chebyshev polynomials in the radial direction and Fourier modes in the azimuthal direction, associated with a third-order semi-implicit time stepping scheme described in Spalart et al. (1991). ${ }^{34}$ The singularity at the origin of the polar coordinate system is avoided by re-defining the radius from -1 to 1 and using an even number of grid points in the radial direction. ${ }^{35}$ Three Sylvester equations are written, respectively, for $\Delta \Psi, \Psi$, and $u_{x}$, associated with homogeneous Dirichlet boundary conditions and are solved using a Fortran code relying on an optimized Sylvester equation solver from the SLICOT numerical library. ${ }^{36}$ The BCs $\Psi=0$ and $u_{x}=0$ at the wall correspond, respectively, to no-penetration and no-slip in the axial direction. The no-slip BC in the azimuthal direction is enforced by adding particular solutions to the streamfunction, following the influence matrix method for linear equations, ${ }^{37}$ such that the azimuthal velocity $u_{\phi}=-\frac{\partial \Psi}{\partial \eta}$ vanishes at the wall. Under deterministic forcing, the $2 \mathrm{D} / 3 \mathrm{C}$ model is reduced to a set of two ordinary differential equations (ODEs) that are solved in MATLAB using spectral methods based on a Chebyshev polynomial expansion.

\section{SIMPLIFIED 2D/3C MODEL WITH DETERMINISTIC FORCING}

We begin by developing a simplified version of the 2D/3C model subject to a steady, deterministic forcing to study momentum transfer between the in-plane and axial 
velocities. The study of optimal disturbance growth in pipe flow by Schmid and Henningson (1994) ${ }^{1}$ demonstrated that the streamwise-constant mode with azimuthal wavenumber $n=1$ is the most amplified based on an energy norm. Thus, we isolate this mode as a candidate perturbation contributing to the blunting of the velocity profile and consider a forcing with only this one mode in the azimuthal direction, namely

$$
N_{\psi}=N(\eta) \sin \phi .
$$

The streamfunction $\Psi(\eta, \phi)$ has the same azimuthal dependence as the forcing since its governing equation is linear i.e., $\Psi=\Psi_{1}(\eta) \sin \phi$. The axial velocity can be written in terms of a mean deviation from laminar $u_{0}$ and a zero-mean perturbation $u_{1} \cos \phi$ corresponding to the linear response of the system to the forcing,

$$
u_{x}(\eta, \phi)=u_{0}(\eta)+u_{1}(\eta) \cos \phi .
$$

The 2D/3C model can be simplified as follows to predict the steady state mean deviation from laminar, $u_{0}$, obtained with the deterministic forcing profile $N(\eta)$ :

$$
\begin{gathered}
\left(\Delta_{\eta}-\frac{1}{\eta}\right)^{2} \Psi_{1}=-0.5 \operatorname{ReN}(\eta), \\
\left(\Delta_{\eta}-\frac{1}{\eta}\right) u_{1}=0.5 \operatorname{Re} \Psi_{1} d_{\eta}\left(U+u_{0}\right), \\
\Delta_{\eta} u_{0}=-0.5 \operatorname{Re}\left(C \eta-d_{\eta}\left(\Psi_{1} u_{1}\right)\right),
\end{gathered}
$$

where $\Delta_{\eta}=\eta d_{\eta \eta}+d_{\eta}$ is the radial derivative component of the 2D Laplacian premultiplied by $\eta$ and $d_{\eta}=\frac{d}{d \eta}$. In order to obtain the simplest model able to capture the blunting of the velocity profile, we can linearize Eq. (7) under the assumption of small amplitude forcing. The resulting model contains only one nonlinear term in one ODE, the other two ODEs being linear. The presence of at least one nonlinear term is required to obtain a change in mean flow since linear models always give the same mean flow as the one used for the linearization.

A simple inspection of Eqs. (5)-(7) leads to the following observations. Conclusions similar to those of Reddy and Ioannou $(2000)^{19}$ on the energy transfer between streamwise constant modes can be recovered, but this time in terms of momentum transfer and for the pipe instead of Couette flow. The mean turbulent mode $u_{0}$ cannot extract momentum from the laminar base flow and is sustained by the nonlinear interaction between the axial velocity perturbation $u_{1}$ and the streamfunction $\Psi_{1}$, i.e., the $d_{\eta}\left(\Psi_{1} u_{1}\right)$ term in Eq. (7). The non-normality of the system manifests itself in the linear coupling between the laminar base flow and the streamfunction which amplifies the disturbances and generates the axial velocity perturbation $u_{1}$ by convection of streamwise momentum (see Eq. (6)). The shape of the streamfunction determines the amount of blunting obtained for a given amplitude coefficient.

In order to advance further analytically, we write the streamfunction profile $\Psi_{1}$ as a Taylor series at the origin, i.e.,

$$
\Psi_{1}=\sum_{i=0}^{\infty} \alpha_{i} \eta^{i}
$$

and set $\alpha_{0}=0$ in order to enforce continuity in the limit of $\eta$ tending to zero, recalling that $\Psi(\eta, \phi)=-\Psi(\eta, \phi+\pi)$. The forcing profile generating $\Psi_{1}$ is given by

$$
N(\eta)=-\frac{1}{R e}\left(\partial_{\eta \eta}+\frac{1}{\eta} \partial_{\eta}-\frac{1}{\eta^{2}}\right)^{2} \Psi_{1}(\eta) .
$$

We rescale the coefficients $\alpha_{i}$ by $\alpha_{1}$, i.e., $\Psi_{1}=\alpha_{1}\left[\eta+\alpha_{2} \eta^{2}\right.$ $\left.+\alpha_{3} \eta^{3}+\alpha_{4} \eta^{4}+\cdots\right]$, and choose $\alpha_{1}$ such that the change in mean flow induced by the forcing has the same amplitude at its maximum as in the experiments of den Toonder and Nieuwstadt (1997) ${ }^{38}$ at the same Reynolds number, in order to facilitate comparison of the results. Note that, depending on the streamfunction profile, the coefficient $\alpha_{1}$ is not necessarily small in which case the linearization of Eq. (6) is no longer justified. In the following, we solve the nonlinear momentum balance for $u_{1}$ (Eq. (6)) regardless of the amplitude of $\alpha_{1}$. A fundamental streamfunction profile $\Psi_{1}=\alpha_{1}(\eta$ $\left.-3 \eta^{3}+2 \eta^{4}\right)$ is obtained by truncating the series expansion to the fourth order term, enforcing the BCs $\Psi_{1}=\frac{d \Psi_{1}}{d \eta}=0$ at the wall, and requiring that the forcing be bounded at the origin.

Streamfunctions given by $\Psi_{1, a}=\Psi_{1}, \Psi_{1, b}=\Psi_{1}^{2}$, and $\Psi_{1, c}=\Psi_{1}^{3}$ were investigated in order to ascertain the ability of such simple functions to capture key aspects of the blunting of the mean profile and to identify the role of the radial streamfunction profile. The amplitude coefficients, $\alpha_{1}$, were chosen such that the same amount of blunting is realized in each case, as described above in terms of the maximum deviation from laminar. The steady-state equations were solved with 64 grid points in the radial direction, and at a Reynolds number of 24600 , matching one of the pipe flow experiments of den Toonder and Nieuwstadt. ${ }^{38}$ A short convergence study showed that this resolution in the radial direction is sufficient since the maximum relative error compared to the solution computed on 192 grid points is less than one percent.

Figure 2 shows the radial forms of the three analytic streamfunctions, $\Psi_{1, a-c}(\eta)$, and the respective resulting variations of the mean deviation from laminar. Streamfunction profiles $\Psi_{1, b}$ and $\Psi_{1, c}$ reach maximum amplitudes of about 0.05 and 0.25 , respectively, in the core of the pipe, while in comparison $\Psi_{1, a}$ is relatively flat with a maximum amplitude of 0.0085 . Despite such a wide variation in streamfunction amplitude between the three cases, the streamwise velocity profiles are remarkably similar. Even the simple streamfunction profile $\Psi_{1, a}$ leads to a "good" blunting of the velocity profile, in the sense that the general features of the mean profile are reproduced. The maxima of the velocity profiles are situated further from the wall compared to the experimental data, ${ }^{38}$ which likely corresponds to the neglect of the influence of the small scales near the wall by the streamwiseconstant model. The results obtained with the simplified 2D/ $3 \mathrm{C}$ model also show that the velocity profile is relatively independent of the radial shape of the forcing and 


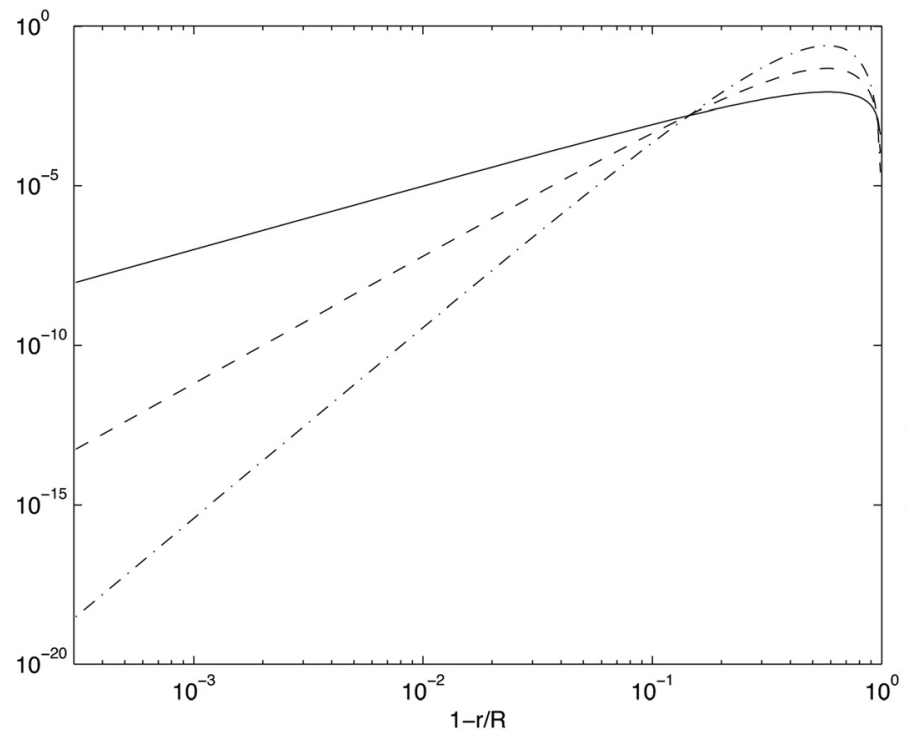

(a)

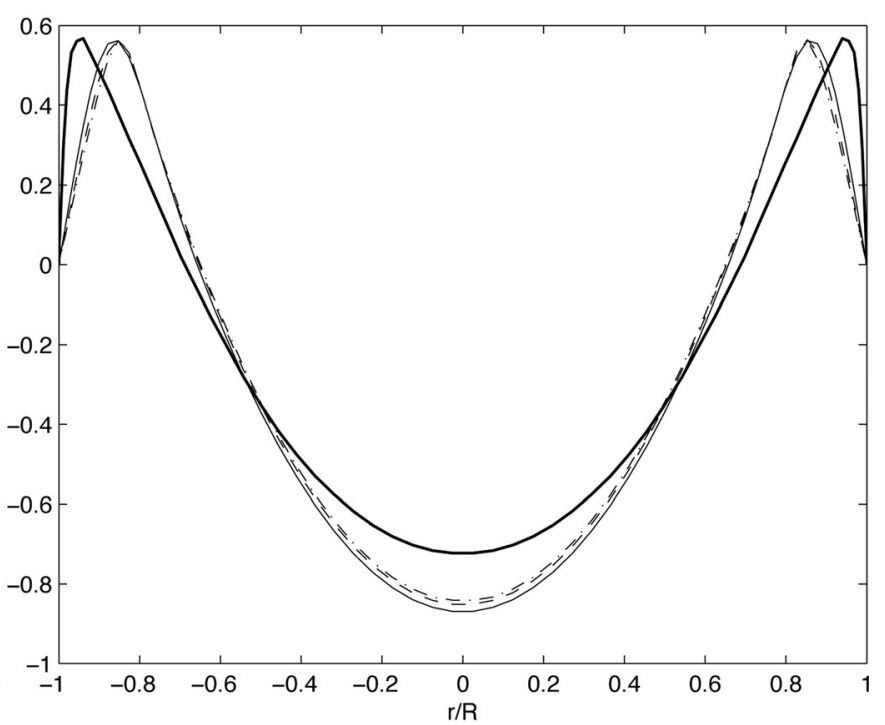

(b)

FIG. 2. (a) Streamfunctions $\Psi_{1, a-c-c}(\eta)$ and (b) corresponding velocity profiles $u_{0}(\eta)$ for $\Psi_{1, a}(\eta)=0.033\left(\eta-3 \eta^{3}+2 \eta^{4}\right) \quad$ (thin $\quad$ solid), $\Psi_{1, b}(\eta)=0.7\left(\eta-3 \eta^{3}+2 \eta^{4}\right)^{2}$ (dashed), and $\Psi_{1, c}(\eta)=14\left(\eta-3 \eta^{3}+2 \eta^{4}\right)^{3}$ (dash-dot) and experimental velocity profile of Ref. 38 at Re $=24600$ (thick solid).

streamfunction, i.e., the profile can be said to be robust to the shape of the streamfunction.

The in-plane kinetic energy, defined as the integral of $\frac{u_{r}^{2}}{2}+\frac{u_{\phi}^{2}}{2}$ over the pipe cross section, varies from $3.7 \times 10^{-4}$ for $\Psi_{1, a}$ to 0.31 for $\Psi_{1, c}$ even though the same amount of blunting is realized by the three streamfunctions $\Psi_{1, a-c}$. This large variation of the in-plane kinetic energy between different streamfunctions (about 3 orders of magnitude) can be understood by consideration of the influence of the near-wall region in the pipe on the overall amplification. Amplification is proportional to the mean shear and surface area, which are both maximum at the wall. Hence, the streamfunction $\Psi_{1, c}$ having a small amplitude near the wall compared to $\Psi_{1, a}$, see Figure 2, does not benefit from the near-wall effects as much as $\Psi_{1, a}$ and needs to reach a larger amplitude in the core (about 30 times larger) in order to give the same amount of blunting as $\Psi_{1, a}$, resulting in a significantly larger inplane kinetic energy.

The large amplitude reached by $\Psi_{1, c}$ in the core of the pipe results in a maximum radial velocity amplitude of $64 \%$ of the bulk velocity compared to $3.3 \%$ for $\Psi_{1, a}$, significantly larger than the radial velocity turbulence intensity measured by den Toonder and Nieuwstadt $(1997)^{38}$ which is about 1 plus unit or equivalently about $5 \%$ of the bulk velocity. A streamfunction that maintains a forcing amplitude comparable to the experimental noise level should, therefore, have a larger amplitude near the wall and relatively constant amplitude over the whole domain. A similar conclusion can be obtained by considering that blunting results from the advection of axial momentum by the radial velocity so that a large amount of blunting is realized when large radial velocities are present. Taking into account that the radial velocity depends on the azimuthal velocity via the continuity constraint, it can be seen that maximization of the ratio $\left|\frac{u_{r}}{u_{\phi}}\right|=\left|\frac{\Psi}{\eta d_{\eta} \Psi}\right|$ suggests that the flattest streamfunction profile (or equivalently the simplest radial dependence) results in the largest amplification. In terms of structures, the largest structures corresponding to the modes with the least zero crossings in the radial direction are more able to redistribute momentum over the cross-section of the pipe. The importance of the modes with the least zero crossings is a known feature of turbulent pipe flow: modes with a radial quantum number of 1 in the study of Duggleby et al. (2007) $)^{39}$ were shown to capture most of the energy in their dynamical eigenfunction decomposition of turbulent pipe flow. Likewise, the singular modes that are most amplified in the study of McKeon and Sharma $(2010)^{40}$ exhibit the lowest number of zero crossings.

Based on our numerical study, the streamfunction $\Psi=0.033\left(\eta-3 \eta^{3}+2 \eta^{4}\right) \sin \phi$ leads to a blunting of the velocity profile whose maximum amplitude matches the experimental data at the same Reynolds number and is generated by a forcing profile $N(\eta)=-90 / R e$ that is constant in the radial direction and whose amplitude over the pipe cross-section is consistent with the experimentally measured rms amplitude of the turbulence fluctuations. The contours of the streamfunction $\Psi=0.033\left(\eta-3 \eta^{3}+2 \eta^{4}\right) \sin \phi$ are plotted on Figure 3 together with a vector plot of the corresponding in-plane velocities and the resulting contours of the axial velocity field. The streamfunction exhibits two counter-rotating rolls which advect the mean shear to create a low- and a high-speed streak of axial velocity defined with respect to the laminar base flow. The highspeed streak sits near the wall whereas the low-speed streak is localized near the centerline. Hence, the flow is on average faster near the wall and slower at the center compared to laminar as is the case for the velocity profile of turbulent pipe flow. The amplification factor between the in-plane and streamwise velocities, defined as the ratio of the extrema, is about 20 in this case. 


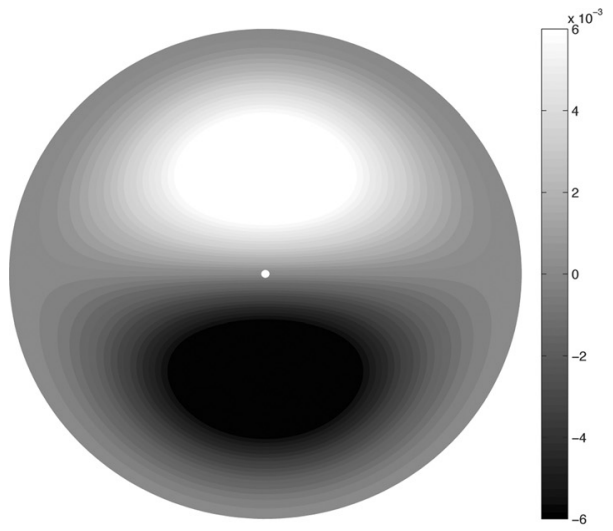

(a)

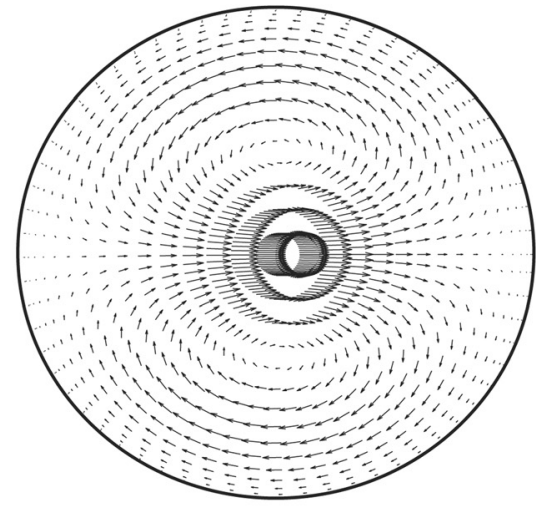

(b)

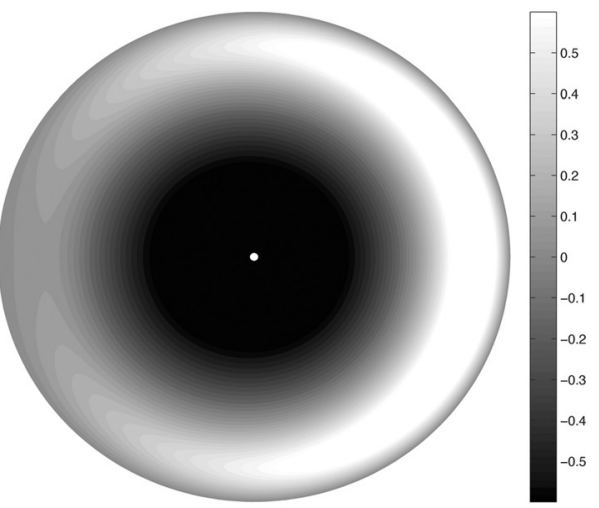

(c)

FIG. 3. Model output for deterministic forcing: (a) contours of the streamfunction $\Psi=0.033\left(\eta-3 \eta^{3}+2 \eta^{4}\right) \sin \phi$, (b) vector plot of the corresponding inplane velocities, and (c) contours of the resulting axial velocity field.

As a final comment related to this simple, steady, deterministic streamfunction analysis, we note that when the azimuthal wavenumber is chosen to match the azimuthal dependence of the spatial puffs observed by Hof et al. $(2004)^{33}$ and a new streamfunction model is derived along the lines described above, the velocity fields produced by our model are remarkably similar to the measured velocity fields near the trailing edge of the puffs. For an azimuthal wavenumber equal to six, the lowest order streamfunction profile that satisfies the $\mathrm{BCs}$ and is generated by a forcing profile bounded at the origin is given by $\Psi_{6}=\alpha_{1}\left(\eta^{4}-2 \eta^{5}+\eta^{6}\right)$; Figure 4 shows the resulting variation of the axial velocity. The wall-normal position of the high- and low-speed axial velocity streaks compares well with Figures 2(e) and 2(f) in the paper by Hof. ${ }^{33}$ Moreover, our model appears to capture the merging of the low-speed streaks and their congregation near the center of the pipe which is observed in experiments ${ }^{33}$ but is not present in the traveling wave solutions of the NSE.

Despite the simplicity of the deterministic streamfunction profiles described in this section, key aspects of the axial velocity variation observed in experiments are recovered. In Sec. IV, we consider a more realistic, time-dependent forcing function in an effort to capture more details associated with the mechanisms of momentum transfer.

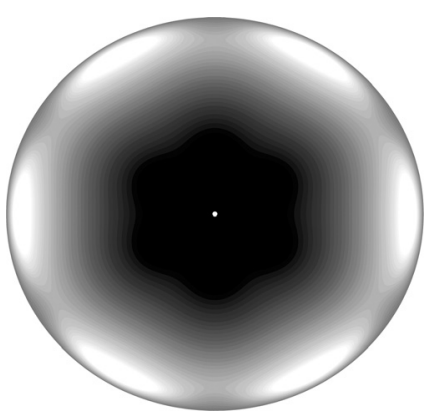

FIG. 4. Contours of the axial velocity induced by the streamfunction $\Psi_{6}(\eta, \phi)=\left(\eta^{4}-2 \eta^{5}+\eta^{6}\right) \sin (6 \phi)$, the light and dark filled contours correspond to regions of the flow, respectively, faster and slower than laminar.

\section{STOCHASTIC FORCING OF THE 2D/3C MODEL}

Effects that are not captured by the (unforced) 2D/3C model and external perturbations that are always present in experiments but not explicitly described by the NSE are likely much better captured by a stochastic forcing than a deterministic one. In this section, we present the results from a simulation of the $2 \mathrm{D} / 3 \mathrm{C}$ model forced by small amplitude white noise, an approach which was successfully explored by Gayme et al. $(2010)^{8}$ in Couette flow as well as several previous linear studies. Such stochastic forcing has the advantage over deterministic forcing of not relying on any assumption regarding the spatial and temporal dependence of the perturbations, such that the resulting velocity field reflects the direction of maximum (assumed dominant) disturbance amplification. The noise, $N_{\psi}$, is applied at each grid point in space and at every time step and follows a normal distribution with zero mean and a variance that depends on the radius such that the variance per surface area is constant. In order to prevent aliasing in the nonlinear coupling terms in the streamwise velocity equation, we truncate the 2D Fourier transform of the forcing term after the lowest two-third wavenumbers, as described in Canuto (2006). ${ }^{41}$

Representative time traces of the centerline velocity for two different Reynolds numbers are reproduced on Figure 5 and show numerous sharp drops, which we identify as the signature of "streamwise-constant puffs," before increasing smoothly nearly back to its laminar value. (Since there is no grid point at the centerline, we approximate the centerline velocity by averaging the axial velocity in the azimuthal direction over the grid points closest to the center of the pipe.) The signatures of the "streamwise-constant puffs" are remarkably similar to the spatial evolution of the centerline velocity from the trailing to the leading edge of the spatial puffs in the numerical simulations of Shimizu and Kida (2009). ${ }^{28}$ We define a puff generation timescale as the time elapsed between two sharp drops of the centerline velocity. At $R e=2200$, the timescale is about 75 dimensionless time units based on the pipe radius and compares well with the time scale of puffs computed from experimental data, ${ }^{42}$ in 


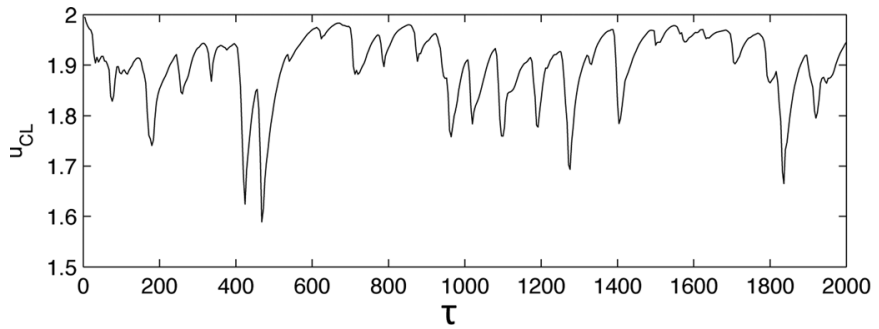

(a)

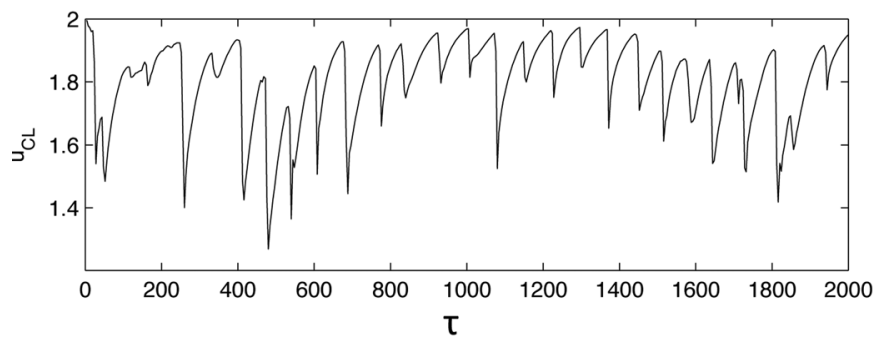

(c)

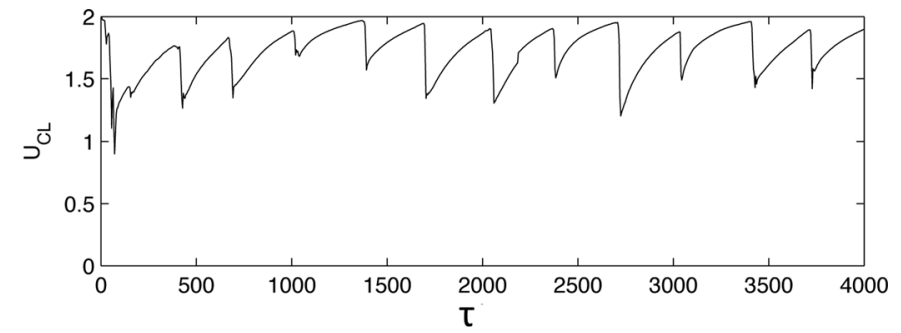

(b)

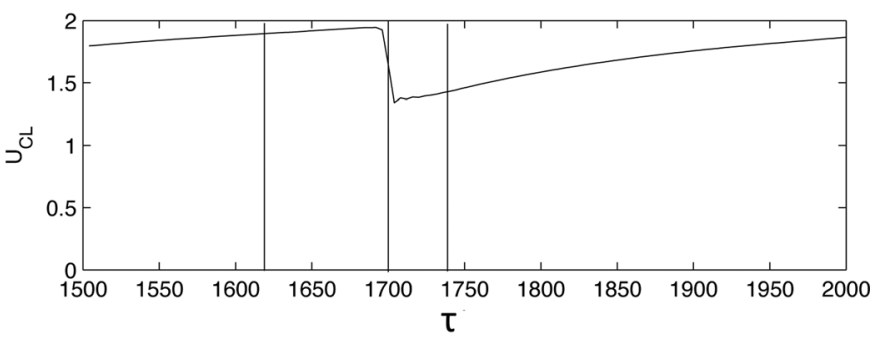

(d)

FIG. 5. Time traces of the centerline velocity from three different simulations, respectively, at $R e=2200$ with 0.0005 and 0.002 rms noise levels (a) and (c) and at $R e=10000$ with $0.002 \mathrm{rms}$ noise level (b). The resolution in the radial direction is $N=48$. (d) Zoom on the time interval during which the samples of Figure 6 are taken. The vertical lines indicate the sampling instants.

which the puffs are 5 to 20 diameters long depending on Reynolds number and convect at nearly the bulk velocity. A puff of length $20 D$ which is separated from the next puff by a laminar region of length equivalent to one puff would lead to a dimensionless timescale based on the pipe radius of 80 .

The puff generation timescale is an increasing function of the Reynolds number, reflecting the fact that puffs tend to be longer in experiments as the Reynolds number increases Figures 5(a) and 5(b), reaching 330 at $R e=10000$, but is relatively independent of the noise amplitude Figures 5(a) and $5(\mathrm{c})$. The drop in centerline velocity associated with the trailing edge of a puff can be observed to be sharper and stronger for larger forcing amplitudes. Note that if we relax the BCs to allow for slip in the azimuthal direction, i.e., we use a shear-stress free condition (not shown), the simulations capture the creation of streamwise vortices and streaks as well as the blunting of the velocity profile, but we do not observe clearly the cyclic generation of puffs in the time evolution of the full velocity field or their signature in the time traces of the centerline velocity. However, for a given forcing amplitude, the amount of blunting realized with the slip BC in the azimuthal direction is larger than with no-slip.

The time evolution of the flow field is characterized by the quasi-periodic generation of "streamwise-constant puffs" followed by their decay and the return of the flow close to the laminar state, i.e., each bursting event is followed by quiescent flow equivalent to the laminar regions that separate the puffs in the experiments of Lindgren. ${ }^{32}$ The three main stages in the evolution of the flowfield corresponding to a "streamwise-constant puff" are plotted on Figure 6 in terms of the axial velocity ((a) to (c)) and the swirling strength ((d) to (f)), defined as the magnitude of the imaginary part of the in-plane velocity gradient eigenvalues and representative of coherent vorticity without the influence of mean shear. These instantaneous fields correspond to the time instants marked with vertical lines in Figure 5(d). During the first stage, patches of swirl move toward the center of the pipe Figures 6(d) and 5(e) and create streaks by convection of the axial momentum Figure 6(a). The radial motion of the coherent swirl corresponds to a lift up of streamwise vortices away from the wall as observed in the simulations of van Doorne and Westerweel (2009) ${ }^{25}$ if we consider the evolution of the vorticity field projected in a plane moving at the bulk velocity. The second stage consists of the segregation of the highand low-speed streaks, the latter being convected toward the center of the pipe resulting in the blunting of the velocity profile characteristic of turbulent pipe flow, Figure 6(b). Once a low-speed streak reaches the center, the centerline velocity drops sharply, as can be seen on the time traces on Figure 5(b). Finally, the swirling strength and streaks decay Figures 6(c) and 6(f) and the flow returns close to the laminar state before the next cycle starts. As the streamwise vortices convect toward the center of the pipe, the sign of the net azimuthal velocity is reversed such that the rotation sign changes from one cycle to the next.

While the output of the $2 \mathrm{D} / 3 \mathrm{C}$ model is a temporal variation of a streamwise constant field, a simplistic comparison with spatial experimental results may be made by assuming an appropriate convection velocity to be the bulk velocity. With this in mind, the time evolution of the velocity field is remarkably similar to the flow visualizations by Hof et al. ${ }^{33}$ in transitioning pipe flow when a puff is observed in a reference frame moving with the bulk velocity. Streak merging in experiments was reported by those authors, who showed that the number of streaks in the cross-section decreases due to their merging as the cross-stream observation plane is moved from the trailing edge to the leading edge of a puff. This streak merging, as well as the segregation of the high- and low-speed streaks observed in the experiments is accurately reproduced by the model, as shown in Figure 5. The time 


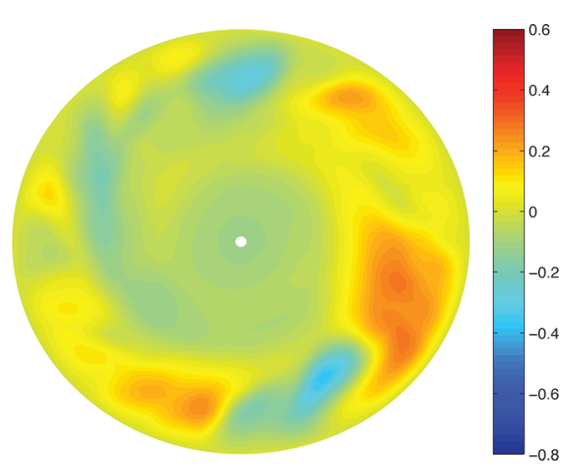

(a)

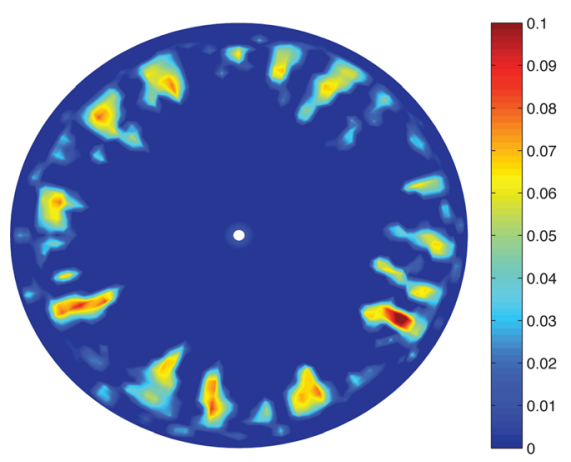

(d)

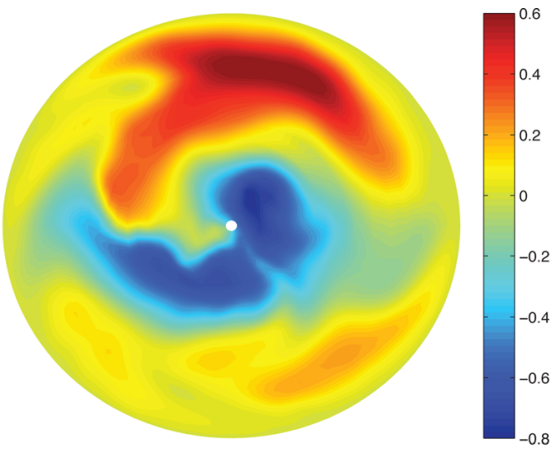

(b)

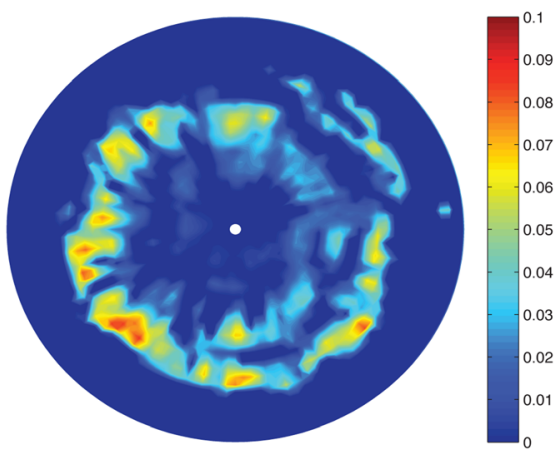

(e)

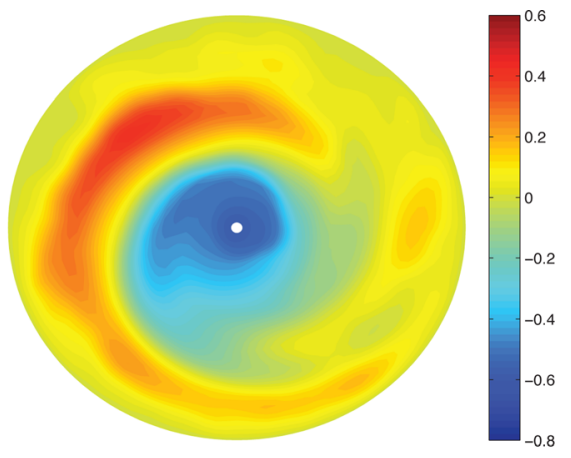

(c)

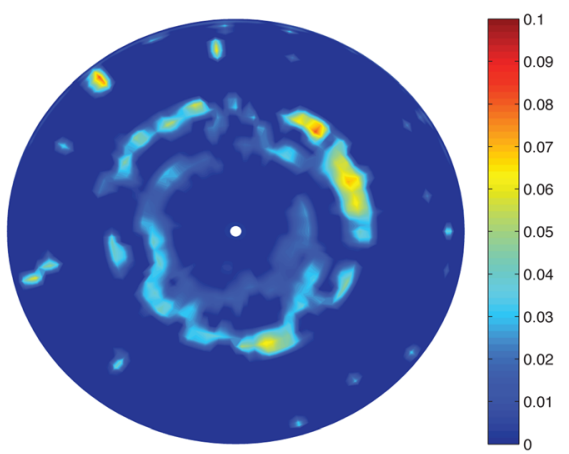

(f)

FIG. 6. (Color online) Contours of the axial velocity, subfigures (a) to (c), and of the swirling strength for the in-plane velocities, subfigures (d) to (f), computed, respectively, at $t=1620, t=1700$, and $t=1740$ dimensionless time units.

evolution of the vorticity field in our simulations is also consistent with the projection of the 3-dimensional vorticity field inside the puff computed by van Doorne and Westerweel ${ }^{25}$ in a plane moving at the bulk velocity, characterized by a lift up of streamwise vortices away from the wall as we move from the trailing to the leading edge of the puff (corresponding to increasing time in our model).

The generation and subsequent decay of the puffs can be described in terms of a simple process driven by background noise, sketched in the inner loop of Figure 7. In essence, the

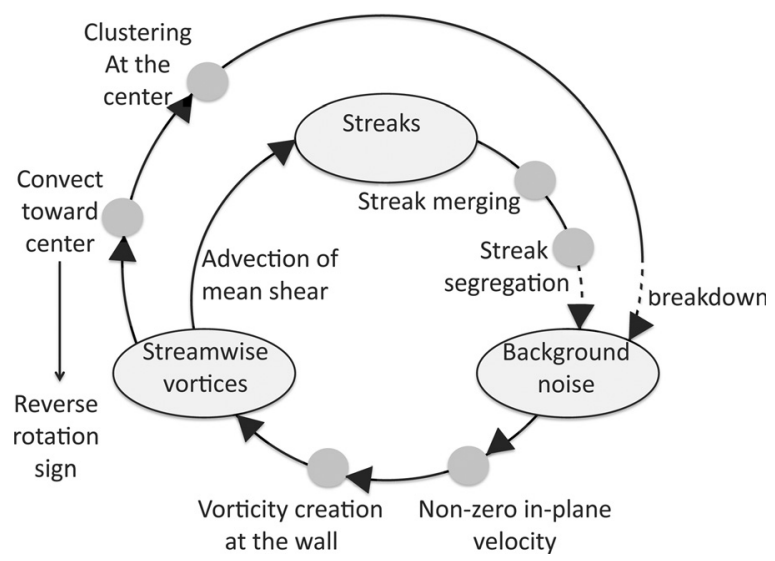

FIG. 7. Diagram detailing the different stages of the QSSP. The dashed lines represent unmodeled effects. presence of background noise in the pipe cross-section results in the formation of streamwise-constant vortices which advect axial momentum to create high- and low-speed streaks of axial velocity. The vortices interact nonlinearly with the streaks to segregate them, i.e., to convect the lowspeed streaks toward the center and the high-speed streaks toward the wall, leading to the blunting of the velocity profile. We term this a "quasi-self-sustaining process" (QSSP) to reflect the fact that, while there is no feedback from the axial velocity to the evolution of the streamfunction (denoted by the dashed line in the inner loop of Figure 7) which could sustain the cycle described in Figure 6, the insertion of small-amplitude stochastic forcing in the cross-stream plane appears to provide an effective replacement for this mechanism. The radial shape of the forcing is directly related to the amount of swirl present in the simulations and, therefore, suggests a method to control the flow by shaping the noise forcing in the spirit of the simple control mechanism developed by Hof et al. ${ }^{29}$ which reduces the inflection points of the velocity profile and leads to a relaminarization of the flow.

A well-studied, fully self-sustaining process has been described for the (3D/3C) NSE by Waleffe $(1997),{ }^{4}$ which relies on the nonlinear interaction of the disturbances developing from the instability of the streaks to force the streamwise vortices. Our results suggest that the blunting of the velocity profile can be considered to be, in essence, a nonlinear, two-dimensional phenomenon in which the directional 
amplification of the $2 \mathrm{D} / 3 \mathrm{C}$ model selects the appropriate disturbance shape from stochastic forcing in order to maintain the QSSP. The model suggests that the sustaining mechanisms are, therefore, insensitive to the type of (nonlinear) interaction invoked to force the streamwise vortices. Based on the simulations presented here, we argue that it is the presence of appropriate, small disturbances in the flow (necessarily contained in the white noise of stochastic forcing), and not their specific interaction that sustains the streamwise vortices and streaks. Hence, the 2D/3C model shows that using unstructured (stochastic) forcing to generate the streamwise vortices appears to be sufficient to capture the formation of the streaks and their segregation resulting in a blunting of the velocity profile and that the overall flow dynamics are relatively insensitive to the particular regeneration process invoked to produce the streamwise vortices. The QSSP is simpler than other processes described in the literature but does appear to capture the minimum turbulence dynamics and produce flow fields dominated by streamwise vortices and streaks whose temporal evolution and topology compares well to experimental visualizations and numerical simulations of puffs, under an appropriate projection onto a plane convecting at the bulk velocity. In addition, our simulations are significantly less computationally intensive than studies of the full $\mathrm{NSE}^{25,28}$ since our domain is $2 D$ and we do not need to track the position of the puffs in time.

Interestingly, our simulations of the stochastically forced 2D/3C model for pipe flow do not reach fully developed turbulence, regardless of the amount of forcing and Reynolds number, even though the same model applied to Couette flow and described in Ref. 8 reached a steady state with a velocity profile in good agreement with the profiles from full 3D simulations at the same Reynolds number, provided that the amount of forcing is appropriately chosen. Mellibovsky et al. ${ }^{43}$ describe pipe flow transition as a twostage process, the first stage consists in the formation of the puffs and the second in their spreading in space. Those authors argue that the two stages are caused by different instability mechanisms. Our simulations support this point of view since we are able to capture the first stage using a streamwise-constant model but not the second stage for which three-dimensional effects are necessary to allow for the puffs to destablize and form slugs which will expand to cover the whole flow domain.

\section{CONCLUSIONS}

We have investigated a (globally stable) streamwiseconstant, $2 \mathrm{D} / 3 \mathrm{C}$ model of turbulent pipe flow subject to two types of very simple forcing profiles, namely a deterministic, steady streamfunction, and stochastic forcing of the streamfunction equation. By retaining (only) the nonlinearity coupling the (cross-plane) streamfunction and the axial velocity, the model permits the study of the physics underlying momentum transfer. This model, which is significantly more tractable than the full Navier-Stokes equations, appears to be capable of capturing key features associated with transition to turbulence, i.e., the blunting of the velocity profile and the generation of streamwise vortices and streaks.
Using the simplest, time-invariant deterministic forcing, we showed that the velocity profile is robust with respect to variations in the forcing profile and we produced realistic velocity fields that are remarkably similar to the flow visualizations by Hof $e t a l .{ }^{33}$ near the trailing edge of a puff. Thanks to the significant reduction in complexity associated with the 2D/3C projection compared to the full Navier-Stokes equations, several observations can be made by examination of the governing equations: momentum is extracted from the laminar base flow by (zero-mean) perturbations via linear nonnormal mechanisms and redistributed via nonlinear interactions of the perturbations to result in a change in mean flow. Hence, our simplistic model allowed us to isolate basic mechanisms leading to the blunting of the velocity profile in pipe flow.

Under stochastic forcing, the model generates "streamwise-constant puffs" at a frequency that depends on the Reynolds number but not on the forcing amplitude. The model captures the first stage of pipe flow transition as described in Ref. 43, i.e., the formation of puffs but not the second stage (spreading of the puffs in space) for which some three-dimensional effects are necessary. The time evolution of the velocity fields produced by our simulations is remarkably similar to flow visualizations in transitioning pipe flow $^{33}$ when a puff is observed in a reference frame moving at the bulk velocity. The segregation of the highand low-speed streaks observed in experiments is accurately captured by the model as well as the streak merging and the lift up of the streamwise vortices away from the wall.

The essential dynamics governing the generation of puffs in pipe flow transition were captured by the streamwiseconstant model and are relatively insensitive to the particular regeneration mechanisms invoked to produce the streamwise vortices, permitting the introduction of a "quasi self-sustaining process" to describe the generation of puffs and the blunting of the mean velocity profile.

\section{ACKNOWLEDGMENTS}

The authors gratefully acknowledge the support of the AFOSR Grant No. FA 9550-09-1-0701 (program manager John Schmisseur).

${ }^{1}$ P. J. Schmid and D. S. Henningson, "Optimal energy density growth in Hagen-Poiseuille flow,” J. Fluid Mech. 277, 197 (1994).

${ }^{2}$ D. S. Henningson and S. C. Reddy, "On the role of linear mechanisms in transition to turbulence," Phys. Fluids 6(3), 1396 (1994).

${ }^{3} \mathrm{~J}$. Kim and J. Lim, "A linear process in wall-bounded turbulent shear flows," Phys. Fluids 12(8),1885 (1993).

${ }^{4} \mathrm{~F}$. Waleffe, "On a self-sustaining process in shear flows," Phys. Fluids 9(4), 883 (1997).

${ }^{5}$ J. S. Bagget, T. A. Driscoll, and L. N. Trefethen, "A mostly linear model of transition to turbulence," Phys. Fluids 7(4), 833 (1995).

${ }^{6}$ D. D. Joseph and L. N. Tao, "Transverse velocity components in fully developed unsteady flows," J. Appl. Mech. 30, 147 (1963).

${ }^{7}$ A. Papachristodoulou, Scalable Analysis of Nonlinear Systems Using Convex Optimization, Ph.D. thesis, California Institute of Technology, 2005.

${ }^{8}$ D. Gayme, B. J. McKeon, A. Papachristodoulou, B. Bamieh, and J. C. Doyle, "A streamwise constant model of turbulence in plane Couette flow,” J. Fluid Mech. 658, 99 (2010).

${ }^{9}$ B. F. Farrell and P. J. Ioannou, "Stochastic forcing of the linearized Navier-Stokes equations," Phys. Fluids 5(11), 2600 (1993). 
${ }^{10} \mathrm{~B}$. Bamieh and M. Dahleh, "Energy amplification in channel flows with stochastic excitation," Phys. Fluids 13(11), 3258 (2001).

${ }^{11} \mathrm{M}$. R. Jovanovic and B. Bamieh, "Componentwise energy amplification in channel flows," J. Fluid Mech. 534, 145 (2005).

${ }^{12}$ B. Eckhardt, "Turbulence transition in pipe flow: Some open questions," Nonlinearity 21, 1 (2008).

${ }^{13}$ K. C. Kim and R. J. Adrian, "Very large-scale motion in the outer layer," Phys. Fluids 11(2), 417 (1999).

${ }^{14}$ J. F. Morrison, W. Jiang, B. J. McKeon, and A. J. Smits, "Scaling of the streamwise velocity component in turbulent pipe flow," J. Fluid Mech. 508, 99 (2004).

${ }^{15}$ M. Guala, S. E. Hommema, and R. J. Adrian, "Large-scale and very-largescale motions in turbulent pipe flow," J. Fluid Mech. 554, 521 (2006).

${ }^{16} \mathrm{~N}$. Hutchins and I. Marusic, "Large-scale influences in near-wall turbulence," Philos. Trans. R. Soc. A 365, 647 (2007).

${ }^{17}$ I. Marusic, B. J. McKeon, P. A. Monkewitz, H. M. Nagib, A. J. Smits, and K. R. Sreenivasan, "Wall-bounded turbulent flows at high Reynolds numbers: Recent advances and key issues," Phys. Fluids 22(6), 1 (2010).

${ }^{18}$ E. Reshotko and A. Tumin, "Spatial theory of optimal disturbances in a circular pipe flow," Phys. Fluids 13(4), 991 (2001).

${ }^{19}$ S. C. Reddy and P. J. Ioannou, "Energy transfer analysis of turbulent plane Couette flow," in Proceedings of the IUTAM Laminar-Turbulent Transition (Springer Verlag, Berlin 2000), pp. 211-216.

${ }^{20}$ H. Salwen, F. W. Cotton, and C. E. Grosch, "Linear stability of Poiseuille flow in a circular pipe," J. Fluid Mech. 98, 273 (1980).

${ }^{21}$ A. Meseguer and L. N. Trefethen, "Linearized pipe flow to Reynolds number $10^{7}$, , J. Comp. Phys. 186, 178 (2003).

${ }^{22}$ A. Meseguer, "Streak breakdown instability in pipe Poiseuille flow," Phys. Fluids 15(5), 1203 (2003).

${ }^{23}$ O. Y. Zikanov, "On the instability of pipe Poiseuille flow," Phys. Fluids 8(11), 2923 (1996).

${ }^{24} \mathrm{~W}$. Schoppa and F. Hussain, "Coherent structure generation in near-wall turbulence," J. Fluid Mech. 453, 57 (2002).

${ }^{25} \mathrm{C}$. W. H. van Doorne and J. Westerweel, "The flow structure of a puff," Philos. Trans. R. Soc. A 367, 489 (2009).

${ }^{26}$ T. M. Schneider, B. Eckhardt, and J. A. Yorke, "Turbulence transition and the edge of chaos in pipe flow," Phys. Rev. Lett. 99(3), (2007).

${ }^{27}$ I. J. Wygnanski and F. H. Champagne, "On transition in a pipe. Part 1: The origin of puffs and slugs and the flow in a turbulent slug," J. Fluid Mech. 59, 281 (1973).
${ }^{28} \mathrm{M}$. Shimizu and S. Kida, "A driving mechanism of a turbulent puff in pipe flow,” Fluid Dynam. Res. 41, 045501 (2009).

${ }^{29}$ B. Hof, A. de Lozar, M. Avila, X. Tu, and T. M. Schneider, "Eliminating turbulence in spatially intermittent flows," Science 327(5972), 1491 (2010).

${ }^{30}$ A. G. Darbyshire and T. Mullin, "Transition to turbulence in constantmass-flux pipe flow," J. Fluid Mech. 289, 83 (1995).

${ }^{31}$ Y. Duguet, A. P. Willis, and R. R. Kerswell, "Slug genesis in cylindrical pipe flow," J. Fluid Mech. 663, 180 (2010).

${ }^{32}$ E. R. Lindgren, "The transition process and other phenomena in viscous flow," Arkiv For Fysik 12, 1 (1957).

${ }^{33}$ B. Hof, C. W. H. van Doorne, J. Westerweel, F. T. M. Nieuwstadt, H. Faisst, B. Eckhardt, H. Wedin, R. R. Kerswell, and F. Waleffe, "Experimental observation of nonlinear traveling waves in turbulent pipe flow," Science 305(5690), 1594 (2004).

${ }^{34}$ P. R. Spalart, R. D. Moser, and M. M. Rogers, "Spectral methods for the Navier-Stokes equations with one infinite and two periodic directions," J. Comp. Phys. 96(2), 297 (1991).

${ }^{35}$ W. Heinrichs, "Spectral collocation schemes on the unit disc," J. Comp. Phys. 199(1), 66 (2004).

${ }^{36}$ I. Jonsson and B. Kagstrom, "RECSY - A high performance library for Sylvester-type matrix equations," in Proceedings of the Euro-Par 2003 Parallel Processing, Vol. 2790, pp. 810-819.

${ }^{37}$ R. Peyret, "Spectral methods for incompressible viscous flow," in Applied Mathematical Sciences (Springer, New York, 2002), Vol. 148.

${ }^{38}$ J. M. J. den Toonder and F. T. M. Nieuwstadt, "Reynolds number effects in a turbulent pipe flow for low to moderate Re," Phys. Fluids 9(11), 3398 (1997).

${ }^{39}$ A. Duggleby, K. S. Ball, M. R. Paul, and P. F. Fischer, "Dynamical eigenfunction decomposition of turbulent pipe flow," J. Turbul. 8(43), 1 (2007).

${ }^{40}$ B. J. McKeon and A. Sharma, "A critical layer framework for turbulent pipe flow,” J. Fluid Mech. 658, 336 (2010).

${ }^{41}$ C. Canuto, Spectral Methods: Fundamentals in Single Domains (Springer, New York, 2006).

${ }^{42}$ M. Nishi, B. Uensal, F. Durst, and G. Biswas, "Laminar-to-turbulent transition of pipe flows through puffs and slugs," J. Fluid Mech. 614, 425 (2008).

${ }^{43}$ F. Mellibovsky, A. Meseguer, T. M. Schneider, and B. Eckhardt, "Transition in localized pipe flow turbulence," Phys. Rev. Lett. 103, (2009). 